\title{
Conflicto armado y feminismo de Estado: la incursión de la problemática de la guerra en la acción pública para las mujeres en Colombia
}

\author{
Carolina Vergel Tovar*
}

\section{RESUMEN}

A partir de una mirada panorámica a la acción pública estatal colombiana relativa a las mujeres, el artículo pretende describir y analizar la forma en la cual la problemática de las mujeres víctimas del conflicto armado entra a hacer parte de las políticas públicas en el país. Además de Ilamar la atención sobre las dificultades para que dicha problemática fuera considerada un problema de interés público, este análisis permite apreciar procesos más amplios de orden institucional y jurídico. Y, más concretamente, aportar al estudio de fenómenos tales como la construcción de un "feminismo de Estado", así como la adopción de una perspectiva de género dentro de las políticas de derechos humanos y de justicia transicional. PALABRAS ClAVE: mujeres, conflicto armado, Estado, políticas públicas, Colombia.

\section{ABSTRACT}

Parting from a panoramic point of view on the Colombian state public policies on women issues, this paper aims to describe and analyze the process through which the problems of women victims of armed conflict became part of public policy in the country. The analysis is also focusing on identifying the difficulties that surrounded the emergence of this problem as a problem of public interest, and therefore allows to appreciate and discuss other processes of institutional and legal order. More specifically, the article looks forward to contribute to the study of the construction of a "State feminism", and also to the discussion concerning the adoption of a gender perspective in human rights policies and transitional justice. KeYwords: Women, armed conflict, State, public policy, Colombia.

* Docente investigadora del Departamento de Derecho Constitucional de la Universidad Externado de Colombia. 


\section{INTRODUCCIÓN}

二 stas reflexiones hacen parte de una investigación doctoral más amplia acerca de C los "Usos militantes e institucionales del derecho a propósito de la causa de las mujeres víctimas del conflicto armado en Colombia". ${ }^{1}$ En líneas generales, dicha investigación buscaba entender el proceso para que la problemática del impacto de la guerra en la vida de las mujeres llegara a ser un asunto de interés público y, específicamente, de interés jurídico. Esta investigación está inscrita en un marco conceptual propio de la sociología del derecho y de los movimientos sociales, así como de los estudios feministas sobre el derecho y la justicia.

En este marco, hemos explorado la forma en la cual el Estado colombiano se planteó por primera vez la pregunta acerca de las mujeres en general y, más concretamente, sobre las mujeres víctimas del conflicto armado en su territorio.

Interesarse en la aparición o surgimiento de un problema o asunto de interés públi$\operatorname{co}^{2}$ puede dar la idea de que es evidente el considerar el rol de la "acción pública"3 en dicho análisis. Sin embargo, la dimensión institucional ha sido un elemento a veces ausente en los llamados estudios socio-jurídicos, en especial en los Estados Unidos. ${ }^{4}$ Esta dimensión institucional implica considerar el rol del Estado y del derecho, según precisa el mismo García Villegas, no solo por acción sino por omisión.

En la investigación adelantada, el rol del Estado se presenta en principio como fundamentalmente indiferente ante la problemática de las mujeres víctimas del conflicto armado y que se manifiesta eventualmente a través de respuestas puntuales. En todo caso, esta cuestión no parece constituir un referencial de la acción pública. ${ }^{5}$

1. Investigación doctoral de orden cualitativo, a partir de un trabajo de campo desarrollado en Bogotá y Medellín, entre 2007 y 2011. Sesenta entrevistas semidirigidas con las personas más importantes y representativas de la causa de las mujeres víctimas del conflicto armado en Colombia, ejercicios de observación etnográfica de espacios e iniciativas de activismo, así como de espacios judiciales, constituyen las principales fuentes de información, fuera del análisis documental.

2. Joseph Gusfield, La Culture des problèmes publics. L'alcool au volant: la production d'un ordre symbolique, traducido por Daniel Cefaï (París: Économica: Coll. Études sociologiques, 2009), 354.

3. Según las escuelas y enfoques, también puede ser entendida como sinónima de "gobernanza" y de "políticas públicas". En todo caso, reenvía al esfuerzo de regulación política en las sociedades contemporáneas y más específicamente, a "la acción colectiva que participa en la creación de un orden social y político, en la dirección de la sociedad y en la regulación de sus tensiones, la integración de grupos sociales y en la resolución de conflictos". P. Lascoumes y P. Legales, Sociologie de l'action publique (París: A. Colin Collection, 2012).

4. Mauricio García Villegas, edit., Sociología jurídica: teoría y sociología del derecho en Estados Unidos (Bogotá: Universidad Nacional de Colombia, 2001), 418.

5. "El 'referencial de la política (...) consiste en un conjunto de requisitos que dan sentido a una agenda pública mediante la definición de criterios de selección y de los métodos de definición de sus objetivos". 
Esta relativa indiferencia se inscribe en una historia más bien compleja de la relación entre el feminismo y el Estado. ${ }^{6}$ A todo lo cual se suman las dificultades específicas del movimiento de mujeres colombiano para articular esfuerzos frente a las ventanas de oportunidad política y jurídica, incluyendo las reformas constitucionales.

Una última consideración conceptual nos parece relevante. Además de determinar los espacios o condiciones de posibilidad para la acción colectiva, considerar la dimensión o marco institucional permite integrar al análisis la cuestión de la responsabilidad del Estado de otra forma: según las tareas que él mismo se ha propuesto. En términos de Berengère Marques-Pereira, conforme a las formas de "responsabilidad pública", es posible comprender de mejor manera las elecciones y justificaciones de los repertorios de acción del activismo. ${ }^{7}$

Es en esta perspectiva que nos parece importante identificar los obstáculos y las oportunidades relacionadas con la adopción de políticas públicas en resonancia con la problemática de las mujeres víctimas del conflicto armado, ${ }^{8}$ con el objetivo de analizar al mismo tiempo, lo que estas nos revelan acerca de las concepciones y consecuencias de la acción pública a propósito de la violencia, el género y las relaciones entre ellos.

Para desarrollar todos los aspectos mencionados, en un primer momento evocaremos muy brevemente los términos del activismo feminista en el momento de la reforma constitucional de 1991, así como sus efectos normativos concretos. En un segundo momento presentaremos las políticas públicas que nos parecen las más importantes para la causa analizada, especialmente aquellas que participan en la construcción de un "feminismo de Estado". .9 Retomamos la definición propuesta por Anne Revillard, para quien se trata de "la acción de las instancias gubernamentales encargadas formalmente de defender el estatus y los derechos de las mujeres". ${ }^{10} \mathrm{Y}$ en un tercer momento

Pierre Muller, "Référentiel", en Laurie Boussaguet y otros, dir., Dictionnaire des politiques publiques (París: Presses de Sciences Po, 2010), 771.

6. Para una visión panorámica de diversas posiciones feministas sobre el Estado, ver Georgina Waylen, "Le genre, le féminisme et l'État: un survol”, en Genre et Politique. Débats et perspectives (París: Gallimard, 2000), 203-32, y 542.

7. Bérengère Marques-Pereira, "L'accès des femmes à l'espace public: du local au national, de l'international au transnational. L'exercice de la responsabilité publique et les rapports de genre en Amérique latine", en Nuevo Mundo Mundos Nuevos (s. 1.: Coloquios, 2008), 4, 21-3.

8. Joyce Gelb y Vivien Hart, "Feminist Politics in a Hostile Environment: Obstacles and Opportunities", en M. Giugni, D. McAdam y C. Tilly, edit., How Social Movements Matter (Minneapolis: University of Minnesota Press, 1999), 149-81.

9. Amy Mazur, Theorizing Feminist Policy (Oxford: Oxford University Press, 2002). Cfr. Jane Jenson y Éléonore Lépinard, "Penser le genre en science politique. Vers une typologie des usages du concept", Revue française de science politique, vol. 59 (2009): 183-201.

10. Anne Revillard, "Entre arène judiciaire et arène législative. Les stratégies juridiques des mouvements féministes au Canada", en Jacques Commaille y Martine Kaluszynski, dir., La fonction politique de la justice (París: La Découverte, 2007), 145-63. 
presentaremos algunos hechos y reflexiones sobre las primeras políticas públicas que se refieren específicamente a las víctimas del conflicto armado.

\section{LAS PRIORIDADES FEMINISTAS ANTE LA OPORTUNIDAD DE REFORMAR LA CONSTITUCIÓN}

El movimiento de mujeres colombiano ha participado activamente de los proyectos de reforma constitucional en la historia reciente. Antes de la reforma de 1991, tuvo lugar un primer proceso participativo, convocado por el gobierno Barco en 1988. ${ }^{11}$ Dicho proceso ha sido objeto de análisis específicos. ${ }^{12}$ En cuanto a la participación de las mujeres, un primer proyecto, elaborado por diecisiete (17) organizaciones, fue presentado al Congreso en ese mismo año. Un segundo proyecto fue propuesto a la Asamblea Nacional Constituyente (ANC) de 1990 y dio lugar a la creación de un colectivo llamado "La mesa mujer y constituyente". Ahora bien, en el momento de organizar las listas de candidaturas de mujeres para la ANC, las tensiones entre quienes preferían una lista independiente, y aquellas que proponían adherir a la lista de un partido político con tendencia de izquierda o de las guerrillas recién desmovilizadas, ${ }^{13}$ terminaron por dividir al movimiento. El precio de dichas divisiones resultó muy caro porque finalmente ninguna de las candidatas resultó electa. ${ }^{14}$

Más que una manifestación de las tensiones entre feministas autónomas y aquellas cercanas a los partidos políticos (como normalmente son esquematizadas incluso en las entrevistas), la cuestión difícil fueron los apoyos potenciales de la causa de las mujeres en el seno de la ANC. Como en otros países de América Latina, la reunión

11. El proyecto fue presentado ante la Comisión III de la Asamblea el 10 de marzo de 1988.

12. Ver por ejemplo Beatriz Quintero, "Las Mujeres Colombianas y la Asamblea Nacional Constituyente de 1991, Participación e Impactos”, en Seminario Internacional: Reformas Constitucionales y Equidad de Género (Santa Cruz de la Sierra: CEPAL/Unidad Mujer y Desarrollo, 2005).

13. "Las primeras desmovilizaciones en Colombia se dieron durante los años noventa cuando nueve grupos guerrilleros se desmovilizaron, dejando a 4.817 individuos acogidos al programa que el gobierno de ese entonces diseñó a través de la Oficina Nacional de Reinserción. Estos grupos fueron el M-19, el Partido Revolucionario de los Trabajadores (PRT), el Ejército Popular de Liberación (EPL), el Movimiento Quintín Lame (MAQL), el Comando Ernesto Rojas (CER), la Corriente de Renovación Socialista (CRS), las Milicias Populares de Medellín (MPM), y el Frente Francisco Garnica y el MIR-COAR", Verdad Abierta, "Desarme y desmovilización”, publicado el 21/08/2008. Disponible en 〈http:/www.verdadabierta.com/ component/content/article/44-procesos-judiciales-justicia-y-paz/137-desmovilizacion-y-desarme->.

14. María Emma Wills, Inclusión sin representación. La irrupción política de las mujeres en Colombia 19702000 (Bogotá: Grupo Editorial Norma, 2007), 398. Julieta Lemaitre Ripoll, El derecho como conjuro (Bogotá: Uniandes/Siglo del Hombre Editores, 2009), 425. 
de la iglesia católica, la izquierda, y los partidos políticos tradicionales, constituyeron un obstáculo de envergadura para el posicionamiento de los intereses de las mujeres. ${ }^{15}$ Según nos explicó una activista que participó del lobbying ante la ANC, ${ }^{16}$ los partidos de izquierda, en especial aquellos recientemente creados por guerrilleros desmovilizados ${ }^{17}$ expresaron su imposibilidad para apoyar las reivindicaciones de las mujeres que pudieran exponerlos a conflictos con la iglesia, siendo esta una de sus principales aliadas para la agenda normativa de paz. ${ }^{18}$

Ahora bien, si se observan los proyectos propuestos por el movimiento de mujeres, así como las normas finalmente adoptadas, y que las mismas activistas presentan como logros de su incidencia, el conflicto armado no figura dentro de las mismas (ver cuadro 1, infra). Fuera de la mención a la objeción de conciencia, agregada al final de la elaboración del proyecto de 1988, no hay mención alguna relativa al conflicto armado. El contenido de los proyectos muestra el lugar prioritario de los derechos sexuales y reproductivos en la agenda feminista del momento, el interés por integrarlos en el derecho positivo, y más concretamente, el reconocimiento de su status constitucional. ${ }^{19}$ Cuestión particularmente importante teniendo en cuenta su naturaleza compleja, que implica libertades y derechos sociales. ${ }^{20}$ Las problemáticas de la violencia y de la participación política son finalmente minoritarias y hay solo una referencia al tema de la exclusión. ${ }^{21}$

15. Elena Garcés y Adriana Marulanda, "Women in Colombia: 'you forge your path as you walk" ", en Janet Mancini Billson y Carolyn Fluehr-Lobban, edit., Female Well-Being. Toward a Global Theory of Social Change (s. 1.: Zed Books, 2005), 133-58.

16. Empleamos el término lobbying en sentido amplio, para denotar las acciones de las activistas de la causa como un "grupo de interés" y por lo mismo, que busca "influenciar los poderes públicos en un sentido favorable a sus intereses", así como ante "otros grupos o la opinión pública en general". Sabine Saurugger, "Groupe d'intérêt", en Dictionnaire des politiques publiques (s. 1.: Presses de Sciences Po, 2010), 309-16.

17. Hasta el momento existía la Unión Patriótica (partido político de izquierda creado, entre otras fuerzas, por desmovilizados de las FARC-EP) y víctima del asesinato selectivo de miles de sus integrantes desde la década de los 80. En el marco de la desmovilización, buena parte de la guerrilla del M-19 conformó el partido político Alianza Democrática M-19. Desmovilizados del EPL, del PRT y del Quintín Lame conformaron otro movimiento político. Estas tres iniciativas políticas fueron las que lograrían representación en la Asamblea Nacional Constituyente. Ver al respecto: Héctor Alonso Moreno Parra, "La Constituyente: un acuerdo político para la paz", en Semanario Virtual Caja de Herramientas. Ed. No. 00255, Semana del 20 al 26 de mayo de 2011. Disponible en 〈http://viva.org.co/cajavirtual/svc0255/articulo06.html〉.

18. Mauricio Romero, "Movilizaciones por la paz, cooperación y sociedad civil en Colombia", en Mauricio Archila, y Mauricio Pardo, edit., Movimientos sociales, Estado y democracia en Colombia (Bogotá: Universidad Nacional de Colombia/Centro de Estudios Sociales/ICANH, 2001), 405-40, 548.

19. D. Lochak llama a este momento el de "consolidación jurídica". Danièle Lochak, Les droits de l'Homme. Que sais-je? (París: La Découverte, 2009), 37-8.

20. Es el caso de la protección de la maternidad, entendida por D. Lochak como "derecho acreencia". Ibíd., 41-3.

21. Para un balance crítico ver Cristina Motta y otras, edit., Observatorio legal de la mujer: el legado de la Constitución (Bogotá: Universidad de los Andes/Facultad de Derecho/Centro de Investigaciones Sociojurídicas CIJUS, 1998). Otros análisis celebran la secularización de las relaciones de género, cfr. Wills, Inclusión sin representación, 249. 


\section{Cuadro 1}

\section{Comparación de los proyectos y logros en materia de reforma constitucional del movimiento de mujeres en Colombia} 222324

\begin{tabular}{|c|c|c|}
\hline $\begin{array}{c}\text { Proyecto - } 1988 \\
\text { (17 organizaciones } \\
\text { de mujeres) }\end{array}$ & $\begin{array}{l}\text { Proyecto de } 1991 \text { - "Mesa mujer } \\
\text { y constituyente" } 23\end{array}$ & $\begin{array}{c}\text { Logros }^{24} \text { - Texto constitucional } \\
\text { aprobado en } 1991\end{array}$ \\
\hline $\begin{array}{l}\text { Los puntos centrales eran: } \\
\text { - El lenguaje incluyente. } \\
\text { - Principio amplio de no dis- } \\
\text { criminación. } \\
\text { - La reproducción humana } \\
\text { como un derecho y una res- } \\
\text { ponsabilidad. Opción libre } \\
\text { de maternidad. } \\
\text { - Crianza de hijo/as: respon- } \\
\text { sabilidad compartida. } \\
\text { - Toda estructura familiar de- } \\
\text { be ser sinónimo de una ac- } \\
\text { ción voluntaria, del respeto, } \\
\text { la solidaridad y de la igual- } \\
\text { dad de derechos y respon- } \\
\text { sabilidades. } \\
\text { - Exigencia de servicios pú- } \\
\text { blicos que aseguren la posi- } \\
\text { bilidad de crianza en igual- } \\
\text { dad de condiciones. } \\
\text { - Reconocimiento de la fun- } \\
\text { ción social del trabajo do- } \\
\text { méstico tanto en la dimen- } \\
\text { sión productiva como repro- } \\
\text { ductiva. } \\
\text { En un encuentro posterior, lla- } \\
\text { mado el "Abrazo amoroso por } \\
\text { la vida", realizado entre el 13 y } \\
\text { el } 15 \text { de octubre de 1990, que } \\
\text { buscó extender el consenso } \\
\text { en torno al proyecto, se agre- } \\
\text { ga la petición de suprimir el } \\
\text { servicio militar obligatorio. }\end{array}$ & $\begin{array}{l}\text { Las propuestas de las mujeres pue- } \\
\text { den resumirse y clasificarse a partir de } \\
\text { las temáticas siguientes: } \\
\text { - Propuestas de principios para el } \\
\text { preámbulo: } \\
\text { - Soberanía popular. } \\
\text { - La separación entre la iglesia y el } \\
\text { Estado. } \\
\text { - La participación ciudadana. } \\
\text { - La autodeterminación. } \\
\text { - Propuesta del uso del lenguaje in- } \\
\text { cluyente. } \\
\text { - La prohibición de todas las formas } \\
\text { de exclusión y discriminación. } \\
\text { - La reproducción humana como un } \\
\text { derecho y una responsabilidad. } \\
\text { - El derecho de las mujeres a decidir } \\
\text { libremente sobre la maternidad. } \\
\text { - La definición de la familia. } \\
\text { - Protección de la maternidad. } \\
\text { - La violencia doméstica y la violen- } \\
\text { cia sexual. } \\
\text { - La posibilidad de acciones positivas. } \\
\text { - Participación igualitaria en los espa- } \\
\text { - Lios de decisión. } \\
\text { - ja libertad de conciencia y la ob- } \\
\text { ficación de la CEDAWonciencia. } \\
\text { tico. } \\
\text { - } \text { meglamentación del trabajo domés- } \\
\text { - }\end{array}$ & $\begin{array}{l}\text { Los artículos } 40,42 \text { y } 43 \text { consagra- } \\
\text { ron los derechos de las mujeres. En } \\
\text { ellos se reconoce: } \\
\text { - La garantía de no ser sometida a } \\
\text { ninguna forma de discriminación. } \\
\text { - Ayuda y protección especial du- } \\
\text { rante el embarazo y la etapa pos- } \\
\text { parto. } \\
\text { - El apoyo estatal a la mujer cabe- } \\
\text { za de hogar. } \\
\text { - La libertad de fundar una familia } \\
\text { por vínculos jurídicos o naturales. } \\
\text { - La protección integral de la fa- } \\
\text { milia por parte del Estado y la } \\
\text { sociedad. Para ello, se abre la } \\
\text { posibilidad de determinar legal- } \\
\text { mente el carácter inembargable } \\
\text { del patrimonio familiar y la parti- } \\
\text { cipación igualitaria en la propie- } \\
\text { dad familiar. Se consagra el ca- } \\
\text { rácter invulnerable del honor, la } \\
\text { dignidad y la intimidad familiares. } \\
\text { - La igualdad de derechos y obli- } \\
\text { gaciones en la vida marital. } \\
\text { - La facultad de sancionar legal- } \\
\text { mente toda forma de violencia } \\
\text { intrafamiliar. } \\
\text { - El derecho de la pareja a decidir } \\
\text { libremente el número de hijo/as. } \\
\text { gún las leyes civiles. } \\
\text { de igualdad en los cargos públi- } \\
\text { cos. }\end{array}$ \\
\hline
\end{tabular}

Fuente: elaboración propia.

22. Wills, Inclusión sin representación, 219-20.

23. Beatriz Quintero, "Las Mujeres Colombianas y la Asamblea Nacional Constituyente de 1991. Participación e Impactos".

24. Olga Amparo Sánchez, "Vindicaciones feministas por una vida libre de violencias para las mujeres", capítulo II (extracto), en Las Violencias contra las mujeres en una sociedad en guerra (Bogotá: s. e.: 2008$), 4$. 
Luego de presentar algunos elementos del contexto en el que surgió, así como los términos generales del marco constitucional colombiano relativo los derechos de las mujeres, resulta pertinente conocer los que consideramos algunos de los rasgos fundamentales del proceso de formación del "feminismo de Estado" en Colombia.

\section{LAS POLÍTICAS PÚBLICAS CONCEBIDAS PARA LAS MUJERES}

En tanto instrumento de la acción gubernamental en Colombia, las políticas públicas no se ocuparon de las problemáticas relativas a las mujeres de forma específica antes de la década de $1980 .{ }^{25} \mathrm{Al}$ contrario de las tendencias de los países llamados "desarrollados", estas políticas comenzaron abordando problemáticas a partir de un ángulo que tenía en cuenta factores sociales como la pobreza. Las cuestiones relativas a la igualdad o a los derechos sexuales y reproductivos, centrales en la agenda del feminismo de la segunda ola (incluyendo el caso colombiano), no son objeto de políticas públicas específicas sino en la década siguiente (ver cuadro 2, infra).

Esta inversión en el orden histórico, que caracterizó de hecho la mayor parte de los procesos de institucionalización de la cuestión de género en América Latina, refleja la influencia de políticas internacionales, dentro de las cuales la causa de las mujeres estaba al servicio de una agenda más amplia: la del "desarrollo" ${ }^{26}$ De hecho, las mujeres campesinas fueron pieza fundamental de los esfuerzos regionales por contener la influencia de la revolución cubana y de los movimientos políticos de izquierda. En resumen, se buscó integrarlas a la economía de mercado, para reforzar el enfoque capitalista en la economía rural, sin cuestionar su lugar en la familia. Magdalena León describe todo este proceso, inaugurado por la "década de la mujer" de Naciones Unidas en 1975, y hasta la década de los 90, como el paso de la "neutralidad" con respecto al género, a la "distensión" de las políticas públicas en la materia. ${ }^{27}$

25. Sobre las primeras políticas públicas en Colombia, ver Rafael Garrido Lopera, "Currie, Lauchlin", en Camilo Calderón, edit., Gran Enciclopedia de Colombia, vol. 10 y 11, Biografias (Bogotá: Círculo de Lectores, 1991). Disponible en 〈http://www.banrepcultural.org/blaavirtual/ayudadetareas/economia/personalidades_internacionales $>$. (Consultado el 14/09/16).

26. Magdalena León y Carmen Diana Deere, "Introducción: Hacia una síntesis de la época", en La mujer y la política agraria en América Latina (Bogotá: Siglo XXI editores/Asociación Colombiana para el Estudio de la Población, 1986), 11-26.

27. Magdalena León, "El género en la política pública de América Latina: neutralidad y distensión”, Análisis Politico, No. 20 (1993): 39-52. 


\section{Cuadro 2 \\ Principales políticas públicas a favor de las mujeres / Autoridad responsable}

\begin{tabular}{|c|l|}
\hline 1984 & Política de la mujer rural (Doc. CONPES 2109, Ministerio de Agricultura). \\
\hline 1992 & $\begin{array}{l}\text { Política integral para las mujeres (Doc. CONPES 2626, Consejería Presidencial para la } \\
\text { Juventud, la Mujer y la Familia). }\end{array}$ \\
\hline 1992 & $\begin{array}{l}\text { La política de salud para las mujeres, mujeres por la salud (Resolución 1531 de 1992, Mi- } \\
\text { nisterio de Salud). }\end{array}$ \\
\hline 1994 & $\begin{array}{l}\text { Políticas para el Desarrollo de la Mujer Rural (Conpes social, Consejería Presidencial para } \\
\text { la Juventud, la Mujer y la Familia). }\end{array}$ \\
\hline 1994 & $\begin{array}{l}\text { Política de igualdad y participación de las mujeres (CONPES 2726, Departamento Nacional } \\
\text { de Planeación). }\end{array}$ \\
\hline 2003 & $\begin{array}{l}\text { Seguimiento y ajustes a la política de 1994 (CONPES 2941, Departamento Nacional de } \\
\text { Planeación). }\end{array}$ \\
\hline 2003 & $\begin{array}{l}\text { Política Nacional de Salud Sexual y Reproductiva (Ministerio de Protección Social). } \\
\text { para la Equidad de la Mujer). }\end{array}$ \\
\hline
\end{tabular}

Fuente: elaboración propia.

Según un análisis muy esquemático de esta serie de políticas, es posible identificar dos grandes momentos: el primero, de reformas legislativas desarticuladas, que se extiende hasta la década de 1980, cuando una política específica para las mujeres es puesta en marcha. El segundo momento comienza con la creación de autoridades propias de un feminismo de Estado, aspecto que expondremos en detalle en el presente análisis.

Antes de ello, cabe agregar que el primer momento reúne políticas puntuales que no buscan repensar el lugar de las mujeres en términos globales. En contraste, ese será un objetivo asignado a las autoridades específicas en la materia. Sin embargo, ese proceso de institucionalización no ha asegurado hasta el momento una transversalización sistemática de la causa de las mujeres y de una perspectiva de género de las políticas públicas en Colombia. El proceso tampoco ha sido lineal y ciertas conquistas institucionales han conocido retrocesos, incluso hasta hoy.

\section{El "FEMinismo de Estado" TOMA FORMA}

En América Latina, la Comisión Interamericana de Derechos Humanos comienza a recomendar la creación de instituciones a favor de las mujeres a partir de los años 
$60 .{ }^{28}$ En Colombia, es en 1990 que se crea la Consejería Presidencial para la Juventud, la Mujer y la Familia, primera autoridad responsable de las políticas públicas para esas diferentes poblaciones, así como de la elaboración de informes para las Naciones Unidas. Dicha Consejería depende directamente de la Presidencia de la República, y más concretamente, de la "Primera Dama" (la esposa del Presidente), con un presupuesto y una estructura específicas. ${ }^{29}$ Las fuentes de financiación fueron el presupuesto nacional y la cooperación internacional (especialmente los fondos del PNUD y de la Agencia de Cooperación Alemana, GTZ). ${ }^{30}$

Con la creación de esta Consejería se lanzó el programa "Mujer prioridad nacional", que buscaba poner en marcha una serie de medidas de ayuda legal a las mujeres, vía las Comisarías de Familia, ${ }^{31}$ apoyar esfuerzos de formación para las mujeres cabeza de familia, medidas de protección social y la institucionalización de la cuestión de "la mujer" en los niveles departamental y local. En el marco de este último objetivo, la Consejería promovió la creación de "oficinas técnicas de la mujer" en diferentes instituciones (ejemplo: en el Ministerio de Agricultura o en los gobiernos departamentales). El programa es considerado por ciertos análisis como un esfuerzo exitoso, ${ }^{32}$ especialmente porque dio lugar a la concepción de la primera política pública integral para las mujeres en 1992 (ver cuadro 2, supra). Otro aspecto resaltado es la participación de la Consejería en las conferencias internacionales organizadas por Naciones Unidas, especialmente del Cairo (1994) y Beijing (1995).

Con la puesta en marcha de esta Consejería y de esta política, el margen de acción para las feministas, según las estructuras de oportunidad política y jurídica ${ }^{33}$ se acrecienta. Al tiempo, las tensiones relativas a la cuestión de la institucionalización se vuelven más visibles. Según el estudio de María Emma Wills, las estrategias adelantadas por las feministas colombianas al respecto pueden clasificarse a partir de dos tendencias: aquellas que privilegian el trabajo directo con las mujeres, y aquellas que

28. Margarita María Peláez Mejía y Luz Stella Rodas Rojas, La política de género en el Estado colombiano: un camino de conquistas sociales (Medellín: Universidad de Antioquia, 2002), 384.

29. Presidencia de la República de Colombia. Decreto 1878 de 1990. Diario Oficial. Año CXXVII. No. 39506, 17 de agosto de 1990, 2.

30. Peláez Mejía y Rodas Rojas, La política de género en el Estado colombiano: un camino de conquistas sociales, 86-7.

31. Procuraduría General de la Nación, "Comisarías de Familia. Línea de Base Nacional", Procurando la Equidad, No. 6, Vigilancia superior a la garantía de los derechos desde la perspectiva de género (Bogotá: LEGIS, 2011), 88.

32. Peláez Mejía y Rodas Rojas, La política de género en el Estado colombiano: un camino de conquistas sociales, 85 .

33. Revillard, "Entre arène judiciaire et arène législative. Les stratégies juridiques des mouvements féministes au Canada". 
optan más bien por incidir en las autoridades públicas. Según este mismo análisis, las últimas son "expresiones feministas que evolucionan hacia una política más activa frente a las instituciones y al derecho". ${ }^{34}$ Norma Villarreal también da cuenta de esas distinciones y clasifica las acciones de las feministas a partir de dos enfoques: el de la "influencia" y el de la "resistencia". ${ }^{35}$ Esta división es además avalada por algunas activistas en entrevista. ${ }^{36}$

Siguiendo el análisis de B. Marques-Pereira acerca de la estructuración de la agenda y la puesta en marcha de políticas públicas en América Latina, así como de su efectividad, ${ }^{37}$ encontramos dichas divisiones demasiado esquemáticas, en tanto no reflejan la complejidad de las interacciones entre activistas, sus organizaciones y el Estado. Para empezar porque "las redes de ONG nacionales y transnacionales en América Latina desarrollan no solamente una actividad de vigilancia del aparato estatal, sino también una actividad de elaboración de la normatividad social y de reflexión acerca de la inclusión y la exclusión social y política en materia de relaciones de género". 38 En este proceso, las formas de participación pueden ser múltiples y no se limitan únicamente a trabajar directamente con los poderes públicos. Estas consideraciones permiten inscribir las iniciativas de las feministas colombianas en dinámicas más amplias, y sobre todo, de trascender una visión binaria de la relación entre activismo y poderes públicos.

Ahora bien, aunque son múltiples las activistas que han trabajado con el Estado (y de hecho, como se verá, varias de las consejeras presidenciales en la materia vienen del activismo), las condiciones de la participación dentro de las autoridades públicas, o más concretamenzte, el hecho de convertirse en una "femócrata", entendida como "las feministas que trabajan para mejorar las políticas y los servicios a favor de las mujeres, en el seno de la burocracia gubernamental", ${ }^{39}$ no es una elección que se traduzca automáticamente en un refuerzo de su capital militante.

Ese fue exactamente el caso en el momento de la creación de la Dirección Nacional para la Equidad de la Mujer (DINEM), ${ }^{40}$ promovida por una senadora del Partido

34. Wills, Inclusión sin representación. La irrupción política de las mujeres en Colombia 1970-2000, 248.

35. Norma Villarreal Méndez, "1991-2008. Las mujeres colombianas entre el estado social de derecho y el conflicto armado", Anuario de Hojas de Warmi, No. 14 (2009): 26.

36. Notas de terreno.

37. Bérengère Marques-Pereira, Petra Meier y David Paternotte, dir., Au-delà et en deça de l'État. Le genre entre dynamiques transnationales et multi-niveaux (Louvain-la-Neuve: Bruylant-Academia, 2010), 203.

38. Ibíd.

39. L. Hancock, edit., "Women's policy interests in the market state", en Women, Public Policy and the State (South Yarra: Macmillan Education, 1999).

40. Presidencia de la República de Colombia. Decreto 1440 de 1995. Diario Oficial. Año CXXXI. No. 41983, 31 de agosto de 1995, 1. 
Liberal Colombiano, con el apoyo de otro grupo de mujeres parlamentarias. La nueva institución detenta un estatus más importante a nivel administrativo que la Consejería que la precedió, así como recursos financieros fijos, determinados por la Ley Orgánica del Presupuesto Nacional. ${ }^{41}$ Una reconocida activista, directora de la ONG denominada Casa de la Mujer, es nombrada como su directora (1996-1998).

Una de las prioridades de la política de la DINEM fue aumentar los espacios de participación para las mujeres, en especial de las mujeres de los sectores populares y marginalizados. Gracias a este objetivo y al apoyo financiero, iniciativas como la Ruta Pacífica de las Mujeres pudieron ver la luz. ${ }^{42}$ Se trata de un aspecto importante en la medida que muestra que uno de los ejemplos más estudiados del activismo "de la resistencia" es en realidad resultado de una política gubernamental.

En términos más globales, la política por la "Equidad y la Participación de las Mujeres", promovida por la DINEM, incorpora tres elementos nuevos a los principios de la acción pública que vale la pena resaltar. El primero es la integración explícita de la "perspectiva de género". Efectivamente, si el término no reemplaza completamente la mención a "la mujer", siempre en singular, como en los programas y políticas precedentes, esta variación conceptual muestra una adecuación del lenguaje a los discursos de los encuentros y programas internacionales sobre el tema. Un segundo elemento importante es el objetivo de instaurar un enfoque transversal, bajo la idea de que la DINEM coordinara las propuestas a integrar en los diferentes programas de todas las otras autoridades públicas. El tercer elemento a subrayar es el recurso a los "derechos de las mujeres" para definir tanto la subordinación como la discriminación. Estos tres elementos son inscritos por ciertos análisis dentro de la "tercera ola" del feminismo. ${ }^{43}$

Dentro del mismo gobierno, el de Ernesto Samper (1994-1998), empieza a gestarse otra institución del feminismo de Estado, gracias a una dinámica similar a aquella detrás de la creación de la DINEM. La iniciativa es de Cecilia López, mujer dedicada a la política y perteneciente al partido liberal, autora del estudio que sirvió de base para la concepción de la Política pública antes mencionada, quien fue nombrada en principio Ministra y luego directora del Departamento Nacional de Planeación(DNP). Es en el seno de este departamento de formulación de políticas públicas que se funda el "Consejo de género". La cohabitación de estas dos entidades ha sido estudiada por M. E. Wills, y puede resumirse como el choque entre los enfoques "bottom-up" 4 de

41. Wills, Inclusión sin representación. La irrupción política de las mujeres en Colombia 1970-2000, 217.

42. Ibíd., 235.

43. Bérengère Marques-Pereira y Roland Pfefferkorn, "Genre, politiques sociales et citoyenneté: enjeux et recompositions Introduction", Cahiers du Genre, No. 2 (2011): 5-19.

44. Nelly del Carmen Suárez, "Políticas de mujer rural en Colombia. Una aproximación analítica desde la perspectiva de género", Agronomía, vol. 13, No. 2 (2005): 77-93. 
la DINEM y "up-to-down" del Consejo de género del DNP, los cuales terminaron por anularse mutuamente..$^{45}$ En todo caso, la importancia otorgada a la causa de las mujeres y el impulso propio de las acciones de la DINEM y del Consejo de género del DNP, dieron lugar a una intensa actividad en el Congreso de la República para la aprobación de reformas legales importantes para las mujeres.

Hasta el momento en que adelantamos nuestro trabajo de campo, la última política general referida a las mujeres fue propuesta por el gobierno de Andrés Pastrana, del partido conservador (1998-2002). Esta política estaba enmarcada dentro de una reforma que transforma la DINEM en la Consejería Presidencial para la Equidad de la Mujer (CPEM) ${ }^{46}$ estructura que se mantiene hasta el día de hoy. ${ }^{47}$ Este cambio implica un retroceso en términos de autonomía presupuestal y de margen de acción dentro del gobierno.

Sin embargo, dentro de la política propuesta por esta nueva Consejería, la problemática de "la mujer y el conflicto armado" figura como una línea de trabajo independiente.$^{48}$ En su diagnóstico sobre la "situación general de las mujeres en Colombia", se encuentra un acápite específico sobre la cuestión, que llama la atención sobre tres grupos de mujeres en particular: las mujeres desplazadas por la violencia, las líderes de organizaciones, y las mujeres "reinsertadas" a la vida civil. ${ }^{49}$

El balance elaborado por la CPEM también señala la ausencia de cifras diferenciadas por sexo en las estadísticas de violaciones a los derechos humanos y el derecho internacional humanitario, denuncia el carácter invisible de esas violencias y se apoya en la "Declaración y el Programa de Acción de Viena", aprobado en la Conferencia Internacional de Derechos Humanos de 1993, para exigir una respuesta prioritaria a las mismas.

Dentro de este marco, la política pública propone buscar recursos de la cooperación internacional para la población desplazada por la violencia, al mismo tiempo que busca asegurar un tratamiento prioritario a las víctimas de violencia sexual y de las

45. Wills, Inclusión sin representación. La irrupción política de las mujeres en Colombia 1970-2000, $218-20$.

46. Departamento Administrativo de la Presidencia de la República de Colombia. Decreto 1182 de 1999. Diario Oficial, No. 43626, 29 de junio de 1999.

47. De hecho, la siguiente política integral de mujer y género será propuesta por el actual gobierno de Juan Manuel Santos, en 2010.

48. A partir de este período, en particular desde el año 2000, Colombia asiste a un recrudecimiento de las acciones armadas propias del conflicto, que conocerá un pico estadístico entre los años 2003 y 2008 . Ver al respecto: C. A. Prieto, C. Rocha e I. Marín, "Seis tesis sobre la evolución reciente del conflicto armado en Colombia". Fundación Ideas para la Paz, 23 de septiembre de 2014. Disponible en «http://www.ideaspaz. org/publications/posts/1053>.

49. DINEM. Consejería Presidencial para la Equidad de la Mujer, "Informe de gestión. Agosto 1998-octubre 1999" (Bogotá, diciembre de 1999), 72. 
personas que viven en las zonas de conflicto. La política propone también la realización de investigaciones para esclarecer las violaciones a los Derechos Humanos (DH) y al Derecho Internacional Humanitario (DIH) en donde las víctimas son mujeres.

El balance de estas iniciativas se resume en el marcado interés por la cuestión del desplazamiento forzado: las recomendaciones de la DINEM son finalmente incluidas en el documento del Consejo Nacional de Política Económica y Social (CONPES) sobre desplazamiento. Se organizó un "Encuentro Nacional de mujeres en situación de desplazamiento", coordinado por la Red Nacional de Mujeres. Y la "perspectiva de género", entendida como las acciones específicas a favor de las mujeres, es incorporada al proyecto piloto de un programa de ayuda a la población desplazada a nivel departamental. ${ }^{50}$

Según dos reconocidas activistas que trabajaron en la $\mathrm{CPEM},{ }^{51}$ si bien se promovieron iniciativas importantes para la causa de las mujeres, esta experiencia fue en cierto sentido decepcionante, en razón de la falta de voluntad política por parte del gobierno para apoyar estos temas como una prioridad. Habría que agregar además que su participación les resultó costosa en su calidad de activistas, en la medida que fue percibida por otras feministas como una legitimación, al menos implícita, del cierre de la DINEM, y en términos más amplios, a la pérdida de importancia de las políticas a favor de las mujeres, mientras que la política militarista (el "Plan Colombia"), ganaba terreno.

Esta tensa disyuntiva entre el activismo, de un lado, y la oportunidad concreta de ocupar un cargo en la "femocracia", del otro, se reactualiza cada vez, independientemente de las particularidades de las trayectorias de las feministas, pero también de las particularidades del gobierno al que se sumen. Según el análisis de M. E. Wills, esto se explica en razón de las tensiones internas del movimiento social de mujeres colombiano, en especial en términos de clase. Estas tensiones se explicarían por la distancia entre las percepciones y las experiencias de las feministas "profesionales" (que pertenecen al ámbito urbano), en contraste con las otras activistas, pertenecientes a otros ámbitos, ${ }^{52}$ principalmente populares y campesinos.

A nuestro juicio, las dificultades de articular el aceptar un puesto de poder y el activismo reflejan más bien un doble obstáculo al que se enfrentan las feministas colombianas. Esto reenvía a ciertas de las condiciones propuestas por Anne Revillard y Laure Bereni para considerar el rol de ciertas "femócratas" como un rol exitoso. Se-

50. Ibíd., 53-4.

51. Entrevista realizada en Sisma Mujer. Bogotá, julio de 2010. Entrevista realizada en su casa, Bogotá, junio de 2008.

52. Wills, Inclusión sin representación. La irrupción politica de las mujeres en Colombia 1970-2000, 211-2. 
gún ellas, a partir de los estudios sobre el feminismo de Estado, es posible cuestionar la distinción entre "insiders" y "outsiders". ${ }^{53}$ Para comenzar, porque existen "feministas defendiendo la causa de las mujeres en los escalones más altos del Estado que pueden haber tenido un estatus de "outsider" (en la medida que) la exclusión del sistema político no depende enteramente de la función del puesto que se ocupe". ${ }^{54} \mathrm{Y}$, según ellas todavía, "bien que se trabaje dentro y por el Estado, (las feministas) han podido cuestionar (a veces de manera radical) la estructura desigual de las relaciones de género, incluyendo aquellas que se encarnan en los discursos y prácticas del Estado". ${ }^{5}$

Volviendo al caso colombiano, nos parece que las activistas feministas se han encontrado siempre en medio de una tensión difícil de superar, que ha convertido su estatus en doblemente problemático. De una parte, está la tensión con respecto a las otras activistas, en donde la calidad de outsider con respecto al sistema político es automáticamente cuestionada, por el simple hecho de aceptar un cargo público. De otra parte, en razón de la falta de apoyo por parte del movimiento, por lo cual, ese mismo estatus de outsider termina por ser demasiado pesado para que ellas puedan vehicular cuestionamientos a los discursos y prácticas estatales de manera más directa y eficaz.

De esta forma, ellas representan una amalgama entre los estatus de outsider e insider, pero sin beneficiarse de las ventajas de ninguno de los dos. Al final de cuentas, la desconfianza con respecto al Estado se impone, visto como un Leviathan que ningún capital activista puede relativizar.

Curiosamente, si los gobiernos de Pastrana y, enseguida, los de Uribe comparten y aseguran la continuidad de la política militar del "Plan Colombia", los enfoques sobre el conflicto armado, y por consiguiente, sobre las víctimas del conflicto, no son los mismos. Sucede lo mismo con el feminismo de Estado. Los gobiernos Uribe no modifican la estructura de la CPEM, pero la política hacia las mujeres le da un lugar marginal a la problemática de las mujeres víctimas de conflicto armado, a pesar de su nombre, en principio prometedor: "Mujeres Constructoras de Paz y Desarrollo". ${ }^{56}$ Es la "mujer empresaria" quien le interesa al gobierno, según un comentario recurrente en las entrevistas con las activistas, quienes critican un referencial que encuentran

53. En especial ver M. F. Katzenstein, Faithful and fearless. Moving feminist protest inside the church and military (Princeton: Princeton University Press, 1998). Citada por Laure Bereni y Anne Revillard, "Un mouvement social paradigmatique?, Ce que le mouvement des femmes fait à la sociologie des mouvements sociaux", Sociétés contemporaines, No. 85 (2012): 17-41.

54. Ibíd.

55. Ibíd.

56. Consejería Presidencial para la Equidad de la Mujer, "Mujeres constructoras de paz y desarrollo. Una política nacional orientada a la paz, la equidad y la igualdad de oportunidades" (Bogotá: Presidencia de la República. Colombia, 2009), 100. 
desenfocado frente a la situación crítica de mujeres que exigirían una atención prioritaria por parte del Estado.

Entre las áreas programáticas" de esta política figura la de "violencias contra las mujeres", dentro de la cual está la violencia doméstica, familiar, conyugal y sexual (todas las cuales son agrupadas finalmente bajo la noción de "violencia de género"), y la violencia "a causa del desplazamiento forzado", pero -hecho curioso- no se hace ninguna mención explícita al conflicto armado, desligando completamente las migraciones forzadas del contexto. ${ }^{57}$ Conviene sin embargo precisar que la omisión de la noción de "conflicto armado" es característica de todo el período Uribe. Efectivamente, la "fórmula de estilo" de los discursos del Presidente de la República y del gobierno en general impone que no se hable de la confrontación armada, mucho menos de una guerra, para hablar de "violencia" y de "terrorismo". ${ }^{58}$ Entonces, más que una ruptura con respecto al feminismo de Estado, es con respecto al conflicto armado que hay un punto de quiebre importante.

No obstante, en lo relativo a la política para las mujeres es importante hacer tres anotaciones. La primera se refiere a la ausencia total de activistas feministas involucradas con la problemática de las mujeres víctimas del conflicto como colaboradoras de la CPEM. No hay insiders. La segunda, es la reducción de la comprensión de la violencia a la dimensión doméstica, incluyendo aquella relativa a la problemática del desplazamiento forzado. Finalmente, las Resoluciones del Consejo de Seguridad en la materia y los programas de cooperación internacional para apoyar su puesta en marcha son los instrumentos que parecen forzar al gobierno a hablar expresamente de la problemática, por lo menos en la política dirigida a las mujeres. Esto se limita sin embargo a estudios de factibilidad ${ }^{59} \mathrm{o}$ a estudios en los cuales el enfoque a partir de la violencia doméstica es central. ${ }^{60}$

De esta forma, la violencia doméstica adquiere un estatus político, al mismo tiempo que la cuestión del conflicto armado se disuelve en la dimensión "privada", en lo

57. Ibíd., 42-51.

58. Consejería Presidencial para los Derechos Humanos, "Observaciones del Estado colombiano al Informe de la Alta Comisionada de las Naciones Unidas para los Derechos Humanos sobre Colombia, 2007”, en Programa Presidencial para los Derechos Humanos y el Derecho Internacional Humanitario. Disponible en 〈http://historico.derechoshumanos.gov.co/Prensa/Comunicados/2008/Paginas/070305a.aspx〉. (Consultado el 14/09/16).

59. Ministerio de Relaciones Exteriores. Seminario "Mujeres, Paz y Seguridad: implementación en Colombia de las Resoluciones del Consejo de Seguridad de Naciones Unidas" (Bogotá: Palacio de San Carlos, 2009).

60. Presidencia de la República, "Violencia de género y conflicto armado. Informe de interventoría de un proyecto de investigación” (Bogotá, 2003), 72. 
que constituye un ejemplo de eso que Susan Moller Okin describe como "la negligencia del género y la perpetuación acrítica de una dicotomía público/doméstico" ${ }^{61}$

En todo caso, la tensión a propósito de la cuestión del conflicto armado durante los gobiernos, Uribe plantea también ciertas iniciativas legislativas.

\section{LAS INICIATIVAS DENTRO}

\section{y en torno al Poder Legislativo}

El Congreso de la República también fue puesto al servicio de la causa de las mujeres por la DINEM. Mientras duraron sus actividades (1996-1999), esta dirección promovió una serie importante de proyectos de ley, algunos convertidos en ley. En esta coyuntura, el Colectivo de Mujeres de Bogotá estuvo muy implicado en la adopción de una de las más importantes, la ley 294 de 1996 "para prevenir, remediar y castigar las violencias en las familias". Esta ley hace eco a la ratificación de la Convención Interamericana Belém do Pará sobre la violencia contra las mujeres (otra conquista legislativa de este período), pero como bien lo indica el título, limitando la comprensión de las violencias al ámbito familiar.

Es en el año 2000 -cuando fue creada la CPEM- que son adoptadas dos nuevas reformas legales promovidas por las feministas. A pesar de las tensiones y de la relación ambivalente de las activistas frente al ejercicio del poder, el movimiento social de mujeres colombiano se movilizó fuertemente para garantizar la presencia de las mujeres en el seno de los poderes públicos. Este objetivo se materializa con la ley 581 de 2000 (la Ley de Cuotas), según la cual, el 30\% de los puestos de dirección de los distintos poderes públicos deben estar ocupados por mujeres. Todo el proceso, debates parlamentarios y el alcance de esta ley son todavía objeto de controversias porque su ejecución deja mucho que desear.

Es a propósito de este proyecto que se consolidó la "Bancada de mujeres" del Congreso (grupo de senadoras y de representantes a la Cámara), ${ }^{62}$ bajo la forma de una "Comisión accidental para el Trabajo por la Equidad de Género, los Derechos Sociales, Políticos, Laborales y la Salud Mental, Sexual y Reproductiva de las Mujeres". ${ }^{63}$

61. Susan Moller Okin, "Le genre, le public et le privé", en T.-H. Ballmer-Cao, V. Mottier y L. Sgier, edit., Genre et politique. Débats et perspectives (París: Gallimard-Folio, s. a.), 345-96.

62. Wills, Inclusión sin representación. La irrupción politica de las mujeres en Colombia 1970-2000, 223.

63. Integrada por todas las mujeres parlamentarias, independientemente de su partido. Se fundamenta en el art. 66 del Reglamento del Congreso, el cual autoriza a las directivas de cada cámara a crear comisiones accidentales. 
La ley 1257 de 2008 (por el derecho de las mujeres a una vida libre de violencias) es presentada por este grupo como una de sus realizaciones.

Esta ley inscribe la cuestión de las violencias dentro del marco de la protección a los derechos humanos de las mujeres, así como una forma de discriminación, a través de una definición que retoma más ampliamente los términos de la Convención Belém do Parà que la precedente. Esto implica un principio de responsabilidad diferente para el Estado, aspecto que fue objeto de tensiones importantes con el gobierno Uribe. Pero fue la mención al "conflicto armado" la que se expuso a la oposición más radical de la parte del poder Ejecutivo. Finalmente, una fórmula un tanto ambigua fue adoptada: el artículo 9 de la ley declara que el gobierno nacional "adoptará las medidas para investigar o sancionar los miembros de la Policía, de las Fuerzas Armadas, de las Fuerzas de seguridad $y$ de otras fuerzas que cometan actos de violencia contra las niñas y mujeres que se encuentren en situación de conflicto por la presencia de actores armados" (Las cursivas me pertenecen). Una mención especial fue incluida con respecto a las mujeres "en situación de desplazamiento" (art. 9.7).

Así las cosas, la inscripción de la violencia de las mujeres en la lógica propia de los derechos humanos implica una politización parcial de la misma. Sin reconocer expresamente la existencia de actores armados ilegales, ni establecer un lazo claro entre el contexto de conflicto armado y las violencias contra mujeres y niñas, igual compromete al Estado a esclarecer y sancionar dichas conductas.

\section{LAS POLÍTICAS DE AYUDA A LAS "VÍCTIMAS"}

La historia del surgimiento de la causa de las mujeres víctimas del conflicto armado se inscribe también dentro de la historia de las políticas públicas. En ese marco, las políticas específicas para las víctimas del conflicto armado son concebidas por primera vez durante la década de 1990.

Pero, a pesar de que sea posible ubicar esta primera mención legal, es más difícil trazar una historia lineal de las normas y políticas que conciernen a "las personas afectadas por el conflicto armado", para retomar los términos utilizados en las discusiones que constituyeron los primeros balbuceos políticos en la materia.

Para analizar este tema, identificamos tres líneas institucionales diferentes en las cuales se hace referencia de alguna manera a poblaciones afectadas por el conflicto armado. Más que una división de un universo presupuesto de "víctimas", estas tres líneas ponen de manifiesto respuestas institucionales poco articuladas. Las víctimas no forman un objeto unificado, a pesar de tener un origen común. Al contrario, las diferentes líneas de respuesta institucional distribuyen los efectos del conflicto por poblaciones afectadas conforme a una lógica más bien de orden coyuntural. 
Una primera línea la constituyen las políticas que asumen la cuestión a partir de un enfoque asistencial o de urgencia. La segunda, concierne las políticas de DH y DIH. ${ }^{64}$ A partir de una descripción muy puntual de algunos de los aspectos más relevantes de cada una de esas líneas, haremos un paralelo entre sus lógicas, las cuales difieren sensiblemente de unas a otras.

Como trataremos de mostrar, esta comparación pone de manifiesto una cuestión transversal en la evolución de este tipo de políticas en Colombia, cuestión que se plantea en razón de la prolongación del conflicto armado y del aumento de las víctimas, a veces de forma exponencial. En efecto, estas circunstancias interpelan las lógicas que determinan las respuestas institucionales a partir de un registro propio a las situaciones de excepción y, por eso mismo, de orden temporal o pasajero, como las políticas para situaciones catastróficas. Estas mismas circunstancias plantean un cuestionamiento similar con respecto a las políticas que, en contraste, han decidido integrar la problemática de las víctimas a las políticas sociales, como si se tratara de una forma de marginalidad entre otras.

Al mismo tiempo, la cuestión de las víctimas interpela la responsabilidad de los actores armados, incluyendo al Estado, y por eso, implica una tensión con respecto a las políticas en el momento en el que una parte de las prioridades se concentra en la solución negociada del conflicto armado. En esta misma línea, la cuestión de las víctimas se convierte en una pregunta molesta, cuando se trata de evaluar el respeto a los DH y el DIH. Finalmente, esta cuestión plantea la dificultad para manejar los múltiples efectos de las violencias, que no son los mismos para todas las víctimas. Como la guerra no golpea de la misma manera, hay lugar entonces a preguntarse si conviene homogenizar todas las víctimas bajo esa categoría general o no, y si se justifica hacer eventualmente distinciones.

\section{Las POlíticas de ayUda a las POBLaCiOnes}

AFECTADAS POR LAS ACCIONES ARMADAS:

EL ENFOQUE ASISTENCIAL

Los primeros esfuerzos institucionales frente a los daños ocasionados por las acciones armadas hacen parte de políticas concebidas para la atención de urgencias y desastres. Además de formalizar el margen de acción de la Cruz Roja Internacional,

64. Habría una tercera línea que reúne las políticas concebidas para una población específica, generalmente (aunque no de manera sistemática), según el tipo de violencia sufrida, especialmente para la población desplazada, que por razones de espacio no desarrollaremos en el presente escrito. 
el "Sistema Nacional de Prevención y Atención de Desastres", ${ }^{65}$ creado en 1988, fue encargado de ayudar a las poblaciones de los municipios asolados por las confrontaciones armadas, así como por los primeros desplazamientos masivos de población.

En 1993 nace el Plan Nacional de Rehabilitación (PNR). ${ }^{66}$ Se trata de un programa especial de la Presidencia de la República, parte de una política de "paz y normalización de las zonas afectadas por los desequilibrios del desarrollo, una débil presencia institucional y los conflictos sociales". El PNR se propone operar a partir de tres estrategias inspiradas de las políticas del Estado central luego de la reforma constitucional de 1991: la promoción de la participación comunitaria, de la descentralización y del desarrollo rural. Se trata también de una política que se focaliza exclusivamente en las zonas rurales. Los "remedios" son concebidos en función de las necesidades territoriales y se materializan en forma de inversiones, infraestructura, y con la puesta en marcha de programas de creación de puestos de trabajo o de créditos agrícolas.

En 1994, el PNR es fusionado posteriormente con otras instituciones, cuando se crea la Red de Solidaridad Social (RSS). ${ }^{67}$ Con un espíritu similar al del PNR, pero concebida también pensando en las zonas urbanas, uno de los objetivos principales de la RSS fue concebir, financiar y administrar los programas de ayuda a "los sectores más pobres de la población colombiana", en materia de trabajo, educación, alimentación, seguridad social, deporte, recreación, cultura e integración de comunidades marginales (art. 2).

La determinación y delimitación de esas poblaciones "pobres", potencialmente beneficiarias, considera causas ligadas a la violencia, así como aspectos económicos, sociales, situaciones de discapacidad, la edad y el sexo, como "la infancia, la juventud, la vejez, la mujer y la familia (sic)" (art. 3.2). La ley prevé expresamente la ayuda a la población desplazada, así como a los grupos rebeldes o las milicias políticas urbanas que se hayan reinsertado a la vida civil, poniendo en pie de igualdad a las poblaciones de víctimas civiles y a las personas desmovilizadas (art. 3.12).

A pesar de que la ley considere los aspectos distintivos entre los eventuales beneficiarios, las medidas de ayuda se caracterizan todas por un enfoque no individualizado, bajo la forma de inversiones globales en el marco de las políticas sociales del

65. Congreso de la República de Colombia. Ley 46 de 1988. Diario Oficial, No. 38559, Santafé de Bogotá, 2 de noviembre de 1988.

66. Presidencia de la República de Colombia. Decreto 2707 de 1993. Diario Oficial, No. 41159, Cartagena de Indias, 30 de diciembre de 1993.

67. Congreso de la República de Colombia. Ley 368 de 1997. Diario Oficial, Año CXXXIII, No. 43037, Santafé de Bogotá, 8 de abril de 1997. 
gobierno. Este enfoque será modificado posteriormente con la puesta en marcha de una política especial para la población desplazada por la violencia.

Otros cambios importantes dentro de esta lógica de asistencia tienen lugar con la ley 418 de 1997. Para comenzar, por primera vez, la cuestión de la ayuda a las víctimas es incluida en una ley relativa a las condiciones de negociaciones de paz y de las políticas de desmovilización, desarme y rehabilitación (DDR). Es de resaltar que la cuestión es definida en la ley como una problemática de protección de los derechos y libertades. Finalmente, la protección y ayuda a las víctimas son aspectos considerados a propósito de su participación en los procesos penales. Es en este marco que la definición de "víctima" se vuelve esencial: "aquellas personas de la población civil que sufren perjuicios en su vida, grave deterioro en su integridad personal y/o bienes, por razón de actos que se susciten en el marco del conflicto armado interno, tales como atentados terroristas, combates, ataques y masacres entre otros" (art. 15).

La ley impone también el registro de las víctimas. Esta tarea es una responsabilidad de los comités locales para la prevención y apoyo en caso de desastres, asimilando una vez más los registros de las políticas de emergencia con las de ayuda social. Este registro es centralizado por la RSS, quien decide finalmente quiénes son beneficiarios de la ayuda, otorgada en virtud de un principio de solidaridad social. Esta ayuda debe ser entregada por la misma RSS en un término no superior a un año luego de los hechos que hayan causado el daño.

Se impone hacer algunas observaciones. Para empezar, esta ley adopta los criterios de generales de la responsabilidad civil en materia de indemnización de perjuicios, en donde la prueba de un daño cierto es esencial. Se trata, en principio, de un enfoque individual, que considera los daños sufridos caso por caso. Si bien no se habla de "reparación", la forma en la que se concreta la ayuda humanitaria implica beneficios también de orden individual. Otro aspecto a destacar es la posibilidad de ser reconocido como beneficiario cuando se ha sufrido un perjuicio a causa de actos violentos "cometidos por motivos políticos o ideológicos", una medida que amplía la consideración de los actos violentos más allá de los hechos estrictamente ligados a las confrontaciones armadas y por consiguiente, más cercanos a la lógica protectora de los $\mathrm{DH}$.

Finalmente, la ley crea el "Programa de Protección a Víctimas, Testigos, Intervinientes en el Proceso y Funcionarios de la Fiscalía", dentro de las medidas para asegurar la eficacia de la justicia. Las personas potencialmente beneficiarias deben ser protegidas para recibir una asistencia social.

Esta ley fue concebida en principio para ser aplicada de manera temporal y, más precisamente, durante los cuatro años del gobierno de turno. Sus términos se explican esencialmente por la esperanza del éxito de la política de diálogos de paz del momento. Su validez será prolongada y será sujeta a reformas hasta la adopción de la nueva política del gobierno Uribe (enmarcada en la ley de "justicia y paz"). 
En términos institucionales, la RSS fue finalmente reemplazada por la Agencia Presidencial para la Acción social y la Cooperación Internacional, ${ }^{68}$ más conocida como "Acción Social". Esta institución cambiará completamente la definición de los términos y los objetivos de las políticas públicas relativas a las víctimas. Además de centralizar los recursos de las ayudas internacionales que dependen de la Presidencia de la República, esta agencia inscribe los programas de ayuda social y de ayuda humanitaria en el marco de las políticas del "Plan Colombia". De esta manera, la recepción de los beneficios de los programas sociales para las poblaciones más desfavorecidas depende de su participación en los programas del gobierno, especialmente el de "Familias en acción" y "Familias guardabosques".

El primero es un programa de ayuda a la escolarización por hogar y de ayuda alimentaria, el segundo un programa de colaboración financiera con familias que ayuden en la erradicación de cultivos ilícitos. El enfoque "familialista" de los programas es más que evidente. Constituyen además un ejemplo de condicional cash transfers (prestaciones sociales condicionadas), uno de los instrumentos privilegiados de las políticas sociales en América Latina, según el análisis de J. Jenson, en los cuales "el pago de prestaciones sociales a los más pobres está efectivamente condicionado al compromiso de las madres a velar por un tratamiento médico y la escolarización de sus hijos". ${ }^{69}$

La puesta en marcha de "Acción Social" conduce también a la creación de un sistema de reparación administrativa. La noción de "víctima" se ve así modificada. En contraste, el conflicto armado desaparece, a pesar de que se mencionen los mismos eventos violentos que en la ley anterior.

La reparación administrativa, atribuida a título "solidario", tiene lugar en los casos en los cuales "una persona muere o sufre una invalidez permanente por pérdida de alguna de sus extremidades". Se fija un monto en salarios mínimos legales. Los trámites se hacen directamente ante "Acción Social", a condición de presentar todas las pruebas. A pesar de ser de carácter administrativo, este trámite se inspira en el régimen probatorio de la responsabilidad civil.

Luego de este sobrevuelo acerca de las políticas públicas con enfoque asistencial, es evidente que la población objetivo es por definición aquella que se considera como la más vulnerable. La cuestión de las víctimas gana progresivamente un lugar particular que se volverá central. Esta cuestión se impone en todo caso más en virtud de la cercanía de las nociones de ayuda humanitaria y ayuda de emergencia, sobre todo

68. Presidencia de la República de Colombia. Decreto 2467. Diario Oficial, 45976, 21 de julio de 2005.

69. Jane Jenson, "Politiques publiques et investissement social: quelles conséquences pour la citoyenneté sociale des femmes?", Cahiers du Genre, No. 2 (2011): 21-43. 
por el hecho de la concepción de las políticas como medidas de orden temporal, ya sea subordinadas a políticas sociales más amplias, o dependientes de las políticas de negociación de paz que buscan resolver así el conflicto.

Por otra parte, la adopción de un enfoque más jurídico de los prejuicios nos parece otro proceso que debe resaltarse. A pesar de que el elemento de la reparación es introducido como una figura de orden administrativo, se estructura a partir de elementos del derecho de la responsabilidad en donde la individualización y la prueba del perjuicio son esenciales. Este enfoque jurídico implica también el establecimiento de criterios de responsabilidad que deben determinar, al menos, el patrimonio obligado a pagar las eventuales reparaciones.

En materia de reconocimiento y protección a las mujeres, los efectos de estos últimos cambios institucionales pueden resumirse así: de un régimen de consideración del género como una situación de vulnerabilidad in abstracto, pasamos a un régimen en donde hay que probar el impacto de la guerra por el hecho de ser mujer.

\section{Algunas Consideraciones}

\section{ACERCA DE LAS POLÍTICAS DE DH y DIH}

Dar cuenta, por lo menos panorámica, de las instituciones y políticas en materia de DH y DIH excede los límites de la presente reflexión. Por eso nos limitamos a recoger los aspectos de nuestro análisis que nos parecen los más relevantes para continuar con el paralelo propuesto.

Para efectos de sintetizar el análisis, identificamos cuatro tipos de políticas. ${ }^{70}$ Estas se estructuran esencialmente en torno a dos criterios: en consideración de violaciones específicas a los DH, o teniendo en cuenta la identificación de una población afectada por el conflicto armado de una forma particular. El primer criterio delimita las políticas a favor de la población desplazada por la violencia, contra el reclutamiento de menores y a favor de las personas víctimas de las minas antipersonales respectivamente. El segundo criterio concierne las medidas dirigidas a las familias de las perso-

70. Aunque el corpus documental analizado es más amplio, presentamos aquí las referencias básicas al respecto: "Plan de Acción para la prevención y atención del desplazamiento forzado", propuesto en el Documento Conpes 3057, aprobado en noviembre de 1999 y complementado en 2001 por el Conpes 3115 de ese mismo año; la ley 387 de 1997, que crea el Sistema Nacional de Atención Integral a la Población Desplazada (SNAIPD), el "Programa Especializado y Modalidades para la Atención a Niños, Niñas y Adolescentes que se Desvinculan de Grupos Armados Organizados al Margen de la Ley", regulado por la Resolución No. 6020, 30/12/2010; el "Programa Presidencial para la Acción Integral contra Minas", definido por la ley 759 de 2002 y reglamentado por el Decreto 2150 de 2007; finalmente, la ley 782 de 2002 de regulación de los programas de Desmovilización, Desarme y Reintegración (DDR) de combatientes. 
nas desmovilizadas, así como la población desmovilizada directamente, considerada a veces como víctimas.

Nos parece que no es necesario entrar en mucho detalle acerca de la puesta en marcha de cada una de estas políticas para resaltar la multiplicidad de nociones de "víctima", así como la variedad de regímenes jurídicos, de eventuales beneficios y sobre todo, de criterios tenidos en cuenta para cada uno de los casos. En resumen, al pasar revista a cada una de estas políticas se constata la explosión o fragmentación de la categoría de víctima.

Finalmente, esta multiplicidad de respuestas institucionales muestra una construcción que delimita quién puede ser visto o considerado como víctima y en razón de cuáles efectos, pero de una forma coyuntural y parcial. Esto puede explicarse por la evolución del conflicto armado, de las reivindicaciones sociales, de las presiones y de las prioridades de las agendas internacionales de cooperación (la lista de factores incluso podría prolongarse). En todo caso, conforme a nuestro análisis, lo que nos importa subrayar es el mensaje difundido en el espacio público de opinión legítima.

En este sentido, identificamos tres aspectos que resuenan luego de la presentación de todas estas políticas:

- Un primer aspecto es la lógica reactiva que caracteriza las políticas de cara a las víctimas. Dentro de estas políticas no se ve un esfuerzo por construir una visión de conjunto del conflicto armado y de sus repercusiones, más bien se trata de tentativas para remediar sus efectos. Es en este marco que la cuestión de la "perspectiva de género" se plantea. Si ciertas leyes han previsto disposiciones específicas para las mujeres, no hay una reflexión fundadora relativa al peso de las desigualdades de género en los diferentes procesos de victimización.

- Al mismo tiempo, la suma de las diferentes respuestas estatales no permite construir al final de cuentas una noción de víctima más universal. Esta sectorización constituye un segundo aspecto importante a señalar, pues no refleja una organización específica de la acción pública, sino que formaliza estatus jurídicos diferentes entre personas implicadas en el conflicto armado en donde los criterios de justificación no son muy claros. Estas distinciones no corresponden a criterios "clásicos" como el de distinción entre civil y combatiente por ejemplo.

- En contraste, estos criterios invitan a construir "identidades" específicas de las víctimas, tercer aspecto que queremos subrayar. Y por este hecho, tanto del lado de los funcionarios, tanto del de las activistas, se habla de personas "desplazadas", "secuestradas", "reclutadas", etc. Esas distinciones además generan eventualmente una competencia entre los diversos tipos de víctimas. Y entre los llamados "pobres históricos" y las víctimas del conflicto armado. 


\section{CONCLUSIÓN}

El análisis propuesto pone de manifiesto la aparición tardía de la problemática de las mujeres víctimas del conflicto armado en Colombia, tanto en los esfuerzos activistas feministas, pero sobre todo, en la acción pública. Dicho de otra forma, en un país determinado y estructurado en función o como consecuencia de las dinámicas de guerra, especialmente en los últimos 60 años, las preocupaciones de resorte institucional que integran la problemática pueden situarse en la segunda mitad de la década de 1990. Y, dentro de este panorama, el espacio menos protagónico al respecto lo constituye el feminismo de Estado.

Estas consideraciones, además de mostrar las dificultades propias de la incidencia feminista en la esfera de la acción pública, y en general para que la problemática se inscribiera en la agenda estatal, permite poner de presente las deficiencias de los primeros esfuerzos institucionales por asumir las consecuencias de la guerra para la vida de las mujeres.

Más concretamente, y como reflexión de cierre, encontramos que, aun cuando la problemática fue finalmente acogida en la acción pública, en todos los procesos, normativos e institucionales, no hubo una construcción de una visión de conjunto del conflicto armado ni de las desigualdades de género en los diferentes procesos de victimización, todo lo cual evidencia el desafío que han representado las complejidades del conflicto armado, en consonancia con la dimensión de género, tanto para el activismo como para la acción pública en Colombia.

\section{BIBLIOGRAFÍA}

Garcés, Elena, y Adriana Marulanda. "Women in Colombia: 'you forge your path as you walk'”. En Janet Mancini Billson y Carolyn Fluehr-Lobban, edit., Female Well-Being. Toward a Global Theory of Social Change. s. 1.: Zed Books, 2005.

García Villegas, Mauricio, edit. Sociología jurídica: teoría y sociología del derecho en Estados Unidos. Bogotá: Universidad Nacional de Colombia, 2001.

Garrido Lopera, Rafael. “Currie, Lauchlin”. En Camilo Calderón, edit., Gran Enciclopedia de Colombia, vol. 10 y 11. Bogotá: Círculo de Lectores, 1991. Disponible en 〈http://www. banrepcultural.org/blaavirtual/ayudadetareas/economia/personalidades_internacionales〉.

Gelb, Joyce, y Vivien Hart. "Feminist Politics in a Hostile Environment: Obstacles and Opportunities". En M. Giugni, D. McAdam y C. Tilly, edit., How Social Movements Matter. Minneapolis: University of Minnesota Press, 1999.

Gusfield, Joseph. La Culture des problèmes publics. L'alcool au volant: la production d'un ordre symbolique, traducido por Daniel Cefaï. París: Économica: Coll. Études sociologiques, 2009. 
Hancock, L., edit. "Women's policy interests in the market state". En Women, Public Policy and the State. South Yarra: Macmillan Education, 1999.

Jenson, Jane. "Politiques publiques et investissement social: quelles conséquences pour la citoyenneté sociale des femmes?". Cahiers du Genre, No. 2 (2011).

Jenson Jane, y Éléonore Lépinard. "Penser le genre en science politique. Vers une typologie des usages du concept". Revue française de science politique, vol. 59 (2009).

Katzenstein, M. F. Faithful and Fearless. Moving Feminist Protest inside the Church and Military. Princeton: Princeton University Press, 1998. Citada por Laure Bereni y Anne Revillard, "Un mouvement social paradigmatique?, Ce que le mouvement des femmes fait à la sociologie des mouvements sociaux". Sociétés contemporaines, No. 85 (2012).

Lascoumes, P., y P. Legales. Sociologie de l'action publique. París: A. Colin Collection, 2012.

Lemaitre Ripoll, Julieta. El derecho como conjuro. Bogotá: Uniandes/Siglo del Hombre Editores, 2009.

León, Magdalena. "El género en la política pública de América Latina: neutralidad y distensión”. Análisis Político, No. 20 (1993).

León, Magdalena, y Carmen Diana Deere. “Introducción: Hacia una síntesis de la época”. En La mujer y la política agraria en América Latina. Bogotá: Siglo XXI Editores/Asociación Colombiana para el Estudio de la Población, 1986.

Lochak, Danièle. Les droits de l'Homme. Que sais-je? París: La Découverte, 2009.

Marques-Pereira, Bérengère. 'L'accès des femmes à l'espace public: du local au national, de l'international au transnational. L'exercice de la responsabilité publique et les rapports de genre en Amérique latine”. En Nuevo Mundo Mundos Nuevos. s. 1.: Coloquios, 2008.

Marques-Pereira, Bérengère, y Roland Pfefferkorn. "Genre, politiques sociales et citoyenneté: enjeux et recompositions Introduction”. Cahiers du Genre, No. 2 (2011).

Marques-Pereira, Bérengère, Petra Meier y David Paternotte, dir. Au-delà et en deça de l'État. Le genre entre dynamiques transnationales et multi-niveaux. Louvain-la-Neuve: BruylantAcademia, 2010.

Mazur, Amy. Theorizing Feminist Policy. Oxford: Oxford University Press, 2002.

Moller Okin, Susan. "Le genre, le public et le privé”. En T.-H. Ballmer-Cao, V. Mottier y L. Sgier, edit., Genre et politique. Débats et perspectives. París: Gallimard-Folio, s. a.

Moreno Parra, Héctor Alonso. "La Constituyente: un acuerdo político para la paz”. Semanario Virtual Caja de Herramientas. Ed. No. 00255, Semana del 20 al 26 de mayo de 2011. Disponible en 〈http://viva.org.co/cajavirtual/svc0255/articulo06.html〉.

Motta, Cristina, y otras, edit. Observatorio legal de la mujer: el legado de la Constitución. Bogotá: Universidad de los Andes/Facultad de Derecho/Centro de Investigaciones Sociojurídicas CIJUS, 1998.

Muller, Pierre. "Référentiel”. En Laurie Boussaguet y otros, dir., Dictionnaire des politiques publiques. París: Presses de Sciences Po, 2010.

Peláez Mejía, Margarita María, y Luz Stella Rodas Rojas. La política de género en el Estado colombiano: un camino de conquistas sociales. Medellín: Universidad de Antioquia, 2002. 
Prieto, C. A., C. Rocha e I. Marín, "Seis tesis sobre la evolución reciente del conflicto armado en Colombia". Fundación Ideas para la Paz, 23 de septiembre de 2014. Disponible en 〈http://www.ideaspaz.org/publications/posts/1053〉.

Quintero, Beatriz. “Las Mujeres Colombianas y la Asamblea Nacional Constituyente de 1991, Participación e Impactos". En Seminario Internacional: Reformas Constitucionales y Equidad de Género. Santa Cruz de la Sierra: CEPAL/Unidad Mujer y Desarrollo, 2005.

Revillard, Anne. "Entre arène judiciaire et arène législative. Les stratégies juridiques des mouvements féministes au Canada". En Jacques Commaille y Martine Kaluszynski, dir., La fonction politique de la justice. París: La Découverte, 2007.

Romero, Mauricio. "Movilizaciones por la paz, cooperación y sociedad civil en Colombia". En Mauricio Archila y Mauricio Pardo, edit., Movimientos sociales, Estado y democracia en Colombia. Bogotá: Universidad Nacional de Colombia/Centro de Estudios Sociales/ ICANH, 2001.

Sánchez, Olga Amparo. "Vindicaciones feministas por una vida libre de violencias para las mujeres", Capítulo II (extracto). En Las Violencias contra las mujeres en una sociedad en guerra. Bogotá: s. e.: 2008.

Saurugger, Sabine. "Groupe d'intérêt". En Dictionnaire des politiques publiques. s. 1.: Presses de Sciences Po, 2010.

Suárez, Nelly del Carmen. "Políticas de mujer rural en Colombia. Una aproximación analítica desde la perspectiva de género". En Agronomía, vol. 13, No. 2 (2005).

Verdad Abierta. "Desarme y desmovilización”, publicado el 21/08/2008. Disponible en «http:// www.verdadabierta.com/component/content/article/44-procesos-judiciales-justicia-ypaz/137-desmovilizacion-y-desarme->.

Villarreal Méndez, Norma. “1991-2008. Las mujeres colombianas entre el estado social de derecho y el conflicto armado". Anuario de Hojas de Warmi, No. 14 (2009).

Waylen, Georgina. “Le genre, le féminisme et l'État: un survol”. En Genre et Politique. Débats et perspectives. París: Gallimard, 2000.

Wills, María Emma. Inclusión sin representación. La irrupción política de las mujeres en Colombia 1970-2000. Bogotá: Grupo Editorial Norma, 2007.

\section{Otros}

Congreso de la República de Colombia. Ley 46 de 1988. Diario Oficial, No. 38559, Santafé de Bogotá, 2 de noviembre de 1988. Presidencia de la República de Colombia. Decreto 2707 de 1993. Diario Oficial, No. 41159, Cartagena de Indias, 30 de diciembre de 1993.

---. Ley 368 de 1997. Diario Oficial, Año CXXXIII, No. 43037, Santafé de Bogotá, 8 de abril de 1997.

Consejería Presidencial para la Equidad de la Mujer. "Mujeres constructoras de paz y desarrollo. Una política nacional orientada a la paz, la equidad y la igualdad de oportunidades". Bogotá: Presidencia de la República. Colombia, 2009. 
Consejería Presidencial para los Derechos Humanos. "Observaciones del Estado colombiano al Informe de la Alta Comisionada de las Naciones Unidas para los Derechos Humanos sobre Colombia, 2007”. En Programa Presidencial para los Derechos Humanos y el Derecho Internacional Humanitario. Disponible en 〈http://historico.derechoshumanos.gov.co/ Prensa/Comunicados/2008/Paginas/070305a.aspx>.

Departamento Administrativo de la Presidencia de la República de Colombia. Decreto 1182 de 1999. Diario Oficial, No. 43626, 29 de junio de 1999.

DINEM. Consejería Presidencial para la Equidad de la Mujer. "Informe de gestión. Agosto 1998-octubre 1999”. Bogotá, diciembre de 1999.

Ministerio de Relaciones Exteriores. Seminario "Mujeres, Paz y Seguridad: implementación en Colombia de las Resoluciones del Consejo de Seguridad de Naciones Unidas". Bogotá: Palacio de San Carlos, 2009.

Presidencia de la República de Colombia. Decreto 1440 de 1995. Diario Oficial. Año CXXXI. No. 41983, 31 de agosto de 1995.

---. Decreto 1878 de 1990. Diario Oficial. Año CXXVII. No. 39506, 17 de agosto de 1990.

---. Decreto 2467 de 2005. Diario Oficial, 45976, 21 de julio de 2005.

Presidencia de la República. "Violencia de género y conflicto armado. Informe de interventoría de un proyecto de investigación”. Bogotá, 2003.

Procuraduría General de la Nación. "Comisarías de Familia. Línea de Base Nacional”. Procurando la Equidad, No. 6, Vigilancia superior a la garantía de los derechos desde la perspectiva de género. Bogotá: LEGIS, 2011. 